Meta

Journal des traducteurs

Translators' Journal

\title{
Dictionnaires, objets du patrimoine culturel : le vocabulaire du commerce dans différentes communautés linguistiques
}

\section{Jeanne Dancette}

Volume 49, numéro 4, décembre 2004

URI : https://id.erudit.org/iderudit/009789ar

DOI : https://doi.org/10.7202/009789ar

Aller au sommaire du numéro

Éditeur(s)

Les Presses de l'Université de Montréal

ISSN

0026-0452 (imprimé)

1492-1421 (numérique)

Découvrir la revue

Citer cet article

Dancette, J. (2004). Dictionnaires, objets du patrimoine culturel : le vocabulaire du commerce dans différentes communautés linguistiques. Meta, 49(4),

910-919. https://doi.org/10.7202/009789ar
Résumé de l'article

Les dictionnaires, comme les monuments, les églises ou les sites archéologiques, sont des lieux privilégiés de la mémoire. Ils constituent, à ce titre, des éléments du patrimoine culturel à léguer aux générations futures. Ouvrage centralisateur d'un savoir, le dictionnaire spécialisé a, lui aussi, non seulement une dimension d'usage mais aussi une dimension symbolique. Cet article discute cette idée en s'appuyant sur le Dictionnaire analytique de la distribution/Analytical Dictionary of Retailing. Dépositaire de la mémoire et annonciateur du futur, il rappelle les usages disparus ou en voie de disparition, et consigne les formes actuelles ou en devenir. Ainsi, les archaïsmes, les néologismes, les connotations nouvelles, les calques et emprunts de l'anglais sont-ils donnés à titre d'exemple pour illustrer notre propos, en français et en espagnol. 


\title{
Dictionnaires, objets du patrimoine culturel: le vocabulaire du commerce dans différentes communautés linguistiques ${ }^{1-2}$
}

\author{
JEANNE DANCETTE \\ Université de Montréal, Montréal, Canada \\ jeanne.dancette@umontreal.ca \\ et Université de Genève, Genève, Suisse \\ jeanne.dancette@eti.unige.ch
}

\begin{abstract}
RÉSUMÉ
Les dictionnaires, comme les monuments, les églises ou les sites archéologiques, sont des lieux privilégiés de la mémoire. Ils constituent, à ce titre, des éléments du patrimoine culturel à léguer aux générations futures. Ouvrage centralisateur d'un savoir, le dictionnaire spécialisé a, lui aussi, non seulement une dimension d'usage mais aussi une dimension symbolique. Cet article discute cette idée en s'appuyant sur le Dictionnaire analytique de la distribution/Analytical Dictionary of Retailing. Dépositaire de la mémoire et annonciateur du futur, il rappelle les usages disparus ou en voie de disparition, et consigne les formes actuelles ou en devenir. Ainsi, les archaïsmes, les néologismes, les connotations nouvelles, les calques et emprunts de l'anglais sont-ils donnés à titre d'exemple pour illustrer notre propos, en français et en espagnol.
\end{abstract}

\begin{abstract}
This article illustrates the well-known idea that dictionaries are depositories of cultural heritage in the same way as monuments, festivals or libraries. As symbolic objects, they are testimonies to historical and cultural realities that need to be perpetuated and transmitted. A contemporary dictionary of retailing is discussed here as encompassing today's and yesterday's realities in different parts of the world. More than just a depository of words, this dictionary accounts for habits, customs and usages described in fullfleged encyclopedic articles. Archaïsms and neologisms, new connotations and socio-linguistic factors penetrate and influence the language of trade, creating a tension between local and international usage, with the inevitable dominance of American English in modern retailing. Examples are given in French, English and Spanish.
\end{abstract}

\section{MOTS-CLÉS/KEYWORDS}

dictionnaires, objet culturel, patrimoine, vocabulaire du commerce

\section{Introduction}

La notion de patrimoine est moderne. Selon le sens encore recensé dans le Larousse de 1970, le patrimoine se définit comme un bien qui vient du père ou de la mère; n'y figure pas encore la notion d'héritage culturel. Au sens moderne, le patrimoine est généralement conçu comme l'ensemble des biens tangibles à léguer aux générations futures: les monuments, les églises, les sites. Mais la notion inclut aussi la transmission de l'intangible, à savoir les marques et les étapes de la production artistique ou intellectuelle, du savoir de métier ou du savoir industriel. Ainsi, les archives, les 
bibliothèques, les fêtes, les festivals, les esquisses et, bien entendu, les dictionnaires font-ils leur entrée dans le patrimoine; leur valeur de témoignage, d'objet symbolique, d'objet d'histoire leur donne cette légitimité.

\section{Dictionnaire, objet culturel par excellence}

\section{Dictionnaire, lieu de mémoire}

Même si l'âge d'or du dictionnaire est, en France du moins, le XVIII ${ }^{\mathrm{e}}$ siècle, l'engouement du public ne cesse de s'amplifier à l'époque contemporaine. Sous toutes les formes et pour tout public: dictionnaires, lexiques, glossaires, les 100 termes de $x, y, z$, les $A$ à $Z$ de $x, y, z$, les quid de toute sorte, illustrés, visuels, les juniors, les senior, etc. L'historien Pierre Nora trouve des éléments d'explication à ce phénomène dans le rapport qu'entretiennent les sociétés d'aujourd'hui avec l'histoire et la mémoire:

La curiosité pour les lieux ${ }^{3}$ où se cristallise et se réfugie la mémoire est liée à ce moment particulier de notre histoire. Moment charnière, où la conscience de la rupture avec le passé se confond avec le sentiment d'une mémoire déchirée; mais où le déchirement réveille encore assez de mémoire pour que puisse se poser le problème de son incarnation. Le sentiment de la continuité devient résiduel à des lieux. Il y a des lieux de mémoire parce qu'il n'y a plus de milieux de mémoire.

Qu'on songe à cette mutilation sans retour qu'a représentée la fin des paysans, cette collectivité mémoire par excellence dont la vogue comme objet d'histoire a coïncidé avec l'apogée de la croissance industrielle. Cet effondrement central de notre mémoire n'est pourtant qu'un exemple. C'est le monde entier qui est entré dans la danse, par le phénomène bien connu de la mondialisation, de la démocratisation, de la massification, de la médiatisation (Nora, 1984: XVII-XVIII).

Ainsi, si la paysannerie traditionnelle disparaît, pour reprendre l'exemple de Nora, les dictionnaires qui en immortalisent les modes et techniques deviennent de précieux objets de patrimoine culturel. De la même façon, en anticipant sur l'objet principal de cet article, la disparition du petit commerce à la faveur de la grande distribution rend-elle nécessaire la remise en mémoire des pratiques anciennes, par le biais des dictionnaires ou autres modes de témoignages. Le roman peut être lieu de mémoire, comme l'illustre admirablement le livre régulièrement réédité d'Émile Zola, Au bonheur des dames, qui relate le bouleversement des formes traditionnelles du commerce avec l'apparition des grands magasins et des grands boulevards sur la scène parisienne. Ce serait donc l'accélération de l'histoire, le basculement rapide dans un passé définitivement mort, la perception de ce qui n'est plus, qui rendent les «lieux de mémoire» indispensables.

\section{Dictionnaire, objet culturel, «trésor»}

Comment le dictionnaire devient-il objet de culture, bien du patrimoine? L'écriture et la publication, dit Alain Rey, permettent de conserver, de reproduire, donc de produire, du sens. Le dictionnaire se distingue à cet égard:

Parmi les recueils où nos civilisations graphiques rangent leurs "paroles gelées ", le plus significatif est peut-être le dictionnaire. Par son découpage selon les signes du lexique, il est plus et mieux qu'un conservatoire, car il distribue le discours d'une culture en 
profondeur, en utilisant la nature même de sa langue. Les mots, les noms forment alors un cadre où l'on peut répartir la vision du monde, l'appréhension du réel telles qu'elles se sont incarnées en un code, le français par exemple, ou plutôt dans ses états successifs, les usages de cette langue. (Rey, 1986: 625)

Dès lors, il n'est guère surprenant de désigner les dictionnaires comme des trésors (thesaurus) ${ }^{4}$. Le dictionnaire, comme le trésor, a valeur d'accumulation: s'y retrouvent les signes nécessaires au savoir, à l'expression des identités, à la communication entre les membres du groupe. Mais il a aussi, comme le trésor, une valeur symbolique. Par exemple, note Rey, le Littré se veut trésor. «Depuis Littré, le dictionnaire - ce type de dictionnaire, littéraire ou du moins culturel, historique et thésaurisant - est devenu l'un des hauts lieux de l'imaginaire collectif» (Rey, 1986: 633).

Enfin, comme le trésor, les richesses du dictionnaire sont enfouies; les retrouver est la récompense ultime d'une quête.

\section{Dictionnaire, lieu de centralisation du savoir}

Le dictionnaire, ouvrage de référence, consigne et codifie le savoir. (Les professeurs de traduction le savent bien quand les étudiants claironnent pour justifier leur choix de traduction «je l'ai trouvé dans le Robert!». Le dictionnaire est inattaquable, il est label de qualité. Il devient l'institution de la connaissance linguistique, langagière et sociale, au point où l'on parle du Littré, du nom du lexicographe Émile Littré, du Larousse, du nom de son premier auteur Pierre Larousse, du Robert, etc.

Comment le dictionnaire acquiert-il cette valeur de transmission du savoir? Tout dictionnaire repose sur un effort global de catégorisation et de conceptualisation. Il présente non pas les réalités du monde mais leur schématisation, telle qu'exprimée par le mot et souvent par la définition, l'exemple et la citation. Cette schématisation correspond aux analyses généralement consensuelles d'une nation, d'un groupe social ou professionnel; et, de ce fait, elle est le reflet des intérêts de la nation ou du groupe, ainsi que le rappel de ses souvenirs et le fondement de son enseignement.

Ouvrage centralisateur du savoir, il doit être relativement exhaustif, à cause de la malédiction du non-dénommé: «celui qui n'est pas dit n'a pas d'existence». Les Inuits n'ont pas de mots pour palmier, mais en ont une dizaine pour nommer les différents types de neige!

\section{Derrière les mots il y a le discours}

La magie du dictionnaire s'explique aussi par le discours qu'il contient dans ou entre les lignes. Comme l'exprime Henri Meschonnic, «On cherche des mots, on trouve le discours. On cherche le discours, on trouve des mots» (1991: 9). Les rédacteurs et les auteurs savent bien faire oublier que ce sont des livres de mots (au sens premier, "Wörterbuch» en allemand). Certes, on y ajoute contenu, culture, représentation des sens et des réalités, citations d'auteur, etc. Extrêmement sélectives, ces informations sont le miroir d'une société. Ainsi, le dictionnaire est un discours de discours.

Le dictionnaire exerce une fascination. On ne le lit pas, on le consulte; les mots ouvrent d'innombrables fenêtres sur le monde. Ce pouvoir s'exerce d'autant que le dictionnaire exprime une continuité culturelle. Certains font œuvre et leur pérennité 
reste assurée, comme le dictionnaire philosophique de Voltaire, ou le dictionnaire allemand des frères Grimm, par ailleurs, collectionneurs de contes.

\section{Vocabulaire du commerce}

Dans cette deuxième partie, nous voulons montrer que, comme tout produit, un dictionnaire s'analyse selon deux dimensions, la dimension d'usage et la dimension symbolique. Ce sont ces dimensions qui en font un objet culturel (il produit du sens) et un lieu de mémoire (il rappelle les usages disparus ou en voie de disparition, et consigne les usages actuels dans la mémoire collective).

\section{Dimension d'usage et dimension symbolique}

La valeur d'usage se mesure au besoin ressenti par les usagers potentiels d'un tel outil, qu'il s'agira de satisfaire. Dans le cas des dictionnaires spécialisés, la valeur d'usage réside dans le recensement et la description des termes à l'intérieur d'un domaine de spécialité. En terminologie, on appelle domaine une structuration des connaissances, un ensemble organisé des concepts qui reflètent une sphère d'activité. Il y a production de sens dans cette organisation conceptuelle. Mais n'oublions pas que le domaine est un point de vue, une interprétation du réel, comme le rappelle Bruno de Bessé:

Les domaines sont déterminés en fonction des visions, des connaissances, des pratiques sociales et des besoins des utilisateurs. Les domaines n'ont pas d'existence par euxmêmes. Ils sont délimités du point de vue du chercheur, de l'ingénieur, du technicien, de l'amateur, du civiliste, du pénaliste, du jungien, du freudien, etc. C'est en fonction d'une perspective, d'un point de vue particulier, d'un cadrage, que sera déterminé le contour d'un espace conceptuel (Bessé, 2000: 187).

L'ouvrage sur lequel s'appuie notre développement est le Dictionnaire analytique de la distribution / Analytical Dictionary of Retailing (Dancette et Réthoré, 2000). À quels usages est-il destiné? Outil langagier et de connaissances, il s'adresse à un public d'étudiants d'écoles de commerce, de professionnels de la distribution et de traducteurs. Les utilisations sont multiples: recherche de mots dans plusieurs langues (anglais, français) $^{5}$, recherche d'explications sur les notions, exemples des réalités dénommées, notes de nature encyclopédique, etc.

La dimension symbolique, quant à elle, tient à l'espace et au temps dans lesquels s'inscrit le dictionnaire. Sans que ce soit son objectif premier - ni même un objectif clairement énoncé -, il offre une interprétation du monde du commerce d'aujourd'hui. Notre époque est traversée par la vague de la mondialisation: les échanges se doivent d'être planétaires; les techniques de vente et de gestion se doivent de répondre aux critères de rentabilité maximale; les formats de magasin se doivent d'opter pour la formule du gigantisme ou, à l'inverse, celle de la personnalisation du service, formules auxquelles correspondent des politiques de prix opposées. Mais elle est aussi marquée par des phénomènes qui n’appartiennent pas à la mondialisation «image d'Épinal»; ces phénomènes sont néanmoins décrits. Les réalités du troc, du petit commerçant, des soldes et braderies figurent également dans le dictionnaire; et des métiers de la vente aujourd'hui disparus (regrattier, poraïer, ailler, etc.) sont 
mentionnés. Ainsi, l'ouvrage met au jour les réalités et les tendances récentes du secteur de la grande distribution, et il les nomme, en les opposant aux formes plus archaïques du commerce traditionnel, aux survivances du passé. Le dictionnaire est ainsi producteur de sens et bâtisseur d'un lieu de mémoire, pour le futur comme pour le passé.

\section{Présentation d'une entrée du Dictionnaire analytique de la distribution / Analytical Dictionary of Retailing}

Comme le montre l'entrée AUCTION (fr.: VENTE AUX ENCHÈRES), les informations de nature diverse sont organisées selon plusieurs champs.

\begin{tabular}{|c|c|}
\hline & AUCTION, AUCTION SALE, SALE BY AUCTION \\
\hline & $\begin{array}{l}\text { VENTE }_{\text {nf }} \text { AUX ENCHÈRES, ENCHÈRE } \\
\text { À L'ENCAN, VENTE }_{\text {nf }} \text { Ȧ LA CRIÉE (Fr.) }\end{array}$ \\
\hline$\cdot$ & Définition: \\
\hline & Vente publique au plus offrant de biens ou de titres de propriété. \\
\hline$\cdot$ & Précisions sémantiques: \\
\hline & $\begin{array}{l}\text { Le commissaire-priseur (auctionee ) procède aux enchères qui se font de vive voix dans une } \\
\text { salle de vente (auction room). Il présente l'article et demande une première offre (bid) ou } \\
\text { annonce lui-même un prix initial minimal. Les acheteurs potentiels font des offres pour } \\
\text { enchérir (to bid, to make a bid), chaque offre étant supérieure à la précédente. Le } \\
\text { commissaire-priseur doit adjuger (to knock down, to strike off) l'article au dernier } \\
\text { enchérisseur (bidder). Si celui-ci n'est pas capable de payer la somme offerte, s'il a fait une } \\
\text { folle enchère (false bidding), l'article sera remis en vente. Si le prix obtenu la deuxième fois } \\
\text { est inférieur à celui de la folle enchère, le fol enchérisseur (false bidder) devra payer la } \\
\text { différence. } \\
\text { Les objets vendus aux enchères sont de nature et d'origine diverses. Il s'agit tant de biens } \\
\text { meubles (bijoux, objets d'art, animaux) que d'immeubles. La vente aux enchères peut être } \\
\text { volontaire ou forcée, par exemple, à la suite d'une faillite ou d'une saisie. } \\
\text { Le gouvernement a aussi recours à la vente aux enchères pour se défaire d'objets volés ou } \\
\text { trouvés non réclamés, de biens saisis à la douane ou de biens en surplus. } \\
\text { La vente aux enchères est parfois utilisée sur le marché des bons du Trésor (treasury } \\
\text { bills), sous le nom d'adjudication des bons du Trésor (treasury bill auction, bill auction }(\hat{E} \text {-U.). }\end{array}$ \\
\hline$\cdot$ & Relations internotionnelles: \\
\hline & $\begin{array}{l}\text { Il existe quelques variantes de la vente aux enchères décrite plus haut. } \\
\text { Lors d'une enchère au rabais (Dutch auction, Chinese auction), ou vente aux sous-enchères, } \\
\text { le prix initial est fixé plus haut que le prix que l'on estime obtenir, pour ensuite être baissé } \\
\text { jusqu'au moment où quelqu'un accepte le prix. Ce type d'enchères est utilisé, entre autres, } \\
\text { dans la vente d'animaux (livestock) et dans la vente de poissons et de produits agricoles dans } \\
\text { le domaine du commerce de gros. } \\
\text { Dans la forme la plus moderne, la vente au cadran (clock auction, clock auction sale), ou } \\
\text { marché au cadran, ce processus est automatisé. Les acheteurs sont assis dans un amphi- } \\
\text { théâtre muni d'un grand cadran où les prix sont affichés. Les prix baissent jusqu'à ce qu'un } \\
\text { acheteur arrête le mécanisme au moyen d'un bouton posé devant lui et fasse ainsi une offre. } \\
\text { Si la marchandise est vendue en lots, le premier offrant peut choisir la quantité qu'il veut, et } \\
\text { le reste sera vendu aux acheteurs subséquents. } \\
\text { Dans la vente sous scellés au plus offrant (sealed bid auction), la vente n'est pas publique, } \\
\text { et les offres sont faites par écrit. L'objet sera adjugé au plus offrant. }\end{array}$ \\
\hline
\end{tabular}


Adjudication 1 (adjudication) est le terme légal pour désigner la mise en vente aux enchères d'un bien. Le terme français désigne aussi la déclaration par laquelle le commissairepriseur vend le bien au plus offrant. Il ne faut pas le confondre avec son homonyme adjudication 2 (tender), qui désigne une soumission dans le cadre d'un appel d'offres. Le terme anglais tender peut aussi renvoyer à une offre d'achat, comme dans les expressions vente par adjudication (sale by tender), ou vente par soumission.

On appelle aussi vente à la criée (hawking), ou criée, la méthode de vente dans les marchés publics où les exposants interpellent les consommateurs.

- Compléments d'information:

Hérodote rapporte la pratique de la vente aux enchères à Babylone dès le vi ${ }^{e}$ siècle av. J.-C. Les Romains recouraient aux enchères dans le commerce régulier, mais aussi dans des cas particuliers (empereurs vendant du mobilier royal pour payer leurs dettes, soldats vendant leur butin de guerre).

L'Hôtel Drouot de Paris est la plus ancienne maison de vente aux enchères (auction house) publique du monde (objets d'art et mobiliers anciens). Notons aussi Sotheby's (É.-U.) et Christie's (G.-B.), fondées à Londres en 1733 et en 1766 respectivement, spécialisées dans les articles de luxe (bijoux, tableaux, etc.).

- Informations linguistiques:

- vendre au plus offrant: to sell to the highest bidder

- offrir un prix, enchérir: to bid on

\section{- Contextes:}

Auctions [...] have traditionally provided a rapid and effective means of disposing of goods, especially perishable products. Auctions are also frequently used to sell products directly to the consumers, especially if the value cannot readily be precisely determined, as is the case of works of art or antiques (Britannica Micropeedia 1991).

Aucune formalité spéciale n'est prescrite dans les enchères de meubles. Mais, dans les adjudications d'immeubles, pour laisser aux intéressés le temps de réfléchir, le Code de procédure civile prescrit l'emploi de bougies pouvant rester allumées une minute environ. L'adjudication ne peut être prononcée qu'après l'extinction successive de trois bougies (Grand dictionnaire encyclopédique Larousse 1987).

(On trouvera en annexe l'entrée espagnole correspondante SUBASTA.)

\section{Un échantillon des mots du Dictionnaire (la macrostructure)}

Le Dictionnaire est lieu de mémoire et constructeur de sens car il nomme et définit les réalités présentes et anciennes. (Dans les exemples qui suivent, nous donnons les termes en français, en anglais et en espagnol.)

\section{Les mots qui nomment les réalités, les techniques et les pratiques nouvelles (néosémie)}

- Nouveaux concepts commerciaux: magasin pilote (pilot store, tienda piloto, almacén piloto), magasin intelligent (smart store, tienda inteligente), magasin minimarge (discount store, almacén de descuento, tienda de descuento), grande surface spécialisée minimarge (category killer, asesino de categoría), hypermarché (hypermarket, superstore, hipermercado), mégacentre commercial (megamall, mega centro comercial)

- Techniques nouvelles: marchandisage (merchandising, mercadeo, comercialización, ou bien le verbe mercadear, ou le mot anglais merchandising directement), rentabilité directe 
du produit (direct product profitability, rentabilidad directa del producto), publipostage (direct-mail advertising, publicidad directa por correo), méthode du premier entré-premier sorti ou méthode de l'épuisement progressif (first-in-first-out, FIFO, primero en entrar, primero en salir, FIFO), frontale (facing, frente), lecteur de codes-barres (barcode reader, lector de código de barras), code EAN (EAN code, código EAN), commerce électronique (electronic commerce, comercio electrónico).

- Pratiques nouvelles: gestion des marques (brand management, administración de marcas, gestión de marcas), vente en porte-à-porte (door-to-door selling, venta de puerta en puerta), brouillage des assortiments (scrambled merchandising, ?), guerre des linéaires (shelf space war, guerra de lineales).

\section{Les mots qui rappellent les réalités anciennes (archaïsmes)}

- Mots qui ont disparu des dictionnaires de la langue courante: guelte (vendor's commission, comisión), barguigner (to bargain, negociar, regatear), chaland ${ }^{6}$ (customer). (Les mots espagnols s'emploient encore aujourd'hui.)

- Mots désignant des petits commerces en voie de disparition ou disparus: mercerie (haberdasher's shop, mercería), modiste (women's hap shop, sombrerera, sombrerería de señoras), chapellerie (men's hat shop, sombrerero, sombrerería de caballeros), bonneterie (hosiery shop), marchand des quatre saisons (fruit and vegetable vendor, verdulero, verdulería), harenger (fishmonger, pescadero), regrattier (salt vendor), poraier (herbs and vegetable vendor, verdulero (légumes), botica, boticario (pharmacie)).

\section{Les mots et leurs connotations}

Le Dictionnaire est également lieu de mémoire car il témoigne de l'évolution du vocabulaire et des phénomènes socioterminologiques. L'exemple le plus frappant de cette évolution langagière est le passage des termes commerce et commerçant à distribution et distributeur qui survient à un moment précis de l'histoire française. Édouard Leclerc lance en 1949 ce qu'il appelle ses «centres de distribution» (qui deviendront les hypermarchés d'aujourd'hui).

L'explication de ce changement terminologique, voire de ce changement de paradigme, est que le terme commerce revêt des connotations très négatives. Certes, les petits commerçants sont encore parfois assimilés aux couches méprisées. On reproche aux couches honnies -voleurs, prostituées, vendeurs de rue, mendiants - [la tendance] à parasiter les classes productives (Mayhew, cité par Alborn, Mirowski 1994: 173). Cette image négative qui entache l'ensemble des petits commerçants non productifs - est confirmée par les auteurs Marc Benoun et Marie-Louise Héliès Hassid, spécialistes du commerce:

Après la seconde guerre (sic) mondiale, le commerce avait en France une image plus négative que jamais. Le commerçant déjà suspect, comme toujours, de ne pas être moral car gagnant de l'argent sans être productif, c'est-à-dire sans participer à la transformation des biens, se voyait, en plus, accusé d'avoir activement participé au marché noir. À la Libération, quand on voulait insulter un individu ou désigner un parvenu, on le traitait de BOF (beurre, œufs et fromage) (Benoun et Héliès-Hassid 1995: 14).

Puis arrive la «grande distribution». Au commerce sclérosé, fautif de goulets d'étranglement s'opposent «ces industriels des temps modernes maîtrisant totalement l'écoulement de grandes quantités de produits» (Benoun et Héliès-Hassid 1995: 13). À la boutique du commerçant «symboliquement représentée par un 
crémier faisant les comptes des clients sur un carnet à souches avec un crayon qu'il dégage du coin de son oreille», s'oppose "un univers hautement technologique où règnent la lecture optique, l'informatique interactive et la manutention automatisée » et «demain la vente électronique, (...) et le prélèvement automatique sur le compte bancaire (...)» (Benoun et Héliès-Hassid 1995: 13-14).

Dans l'imaginaire populaire, cet univers ne peut pas être malhonnête ou mesquin et, du même coup, il devient noble et productif. De fait, le vocable distribution, choisi à très bon escient, est associé à de nobles activités (on distribue ses richesses, ses royaumes, la communion, l'aumône, etc.). Il doit évoquer l'image des formes modernes de la vente (productive, qui contribue au produit intérieur brut, qui n'est pas parasite, qui a des pratiques loyales et transparentes, etc.). (On trouvera dans Dancette et Réthoré 1997 une analyse détaillée de cette question.)

Outre les facteurs culturels ou historiques, le vocabulaire commercial suit une évolution déterminée par des critères sociolinguistiques précis.

\section{La plus ou moins grande résistance à l'anglo-américain}

La marée de l'américanisme déferle sur le français comme sur toutes les langues du monde. Les emprunts et les calques en sont les deux manifestations.

- Les emprunts indiquent l'alignement sur l'anglo-américain. Ils sont extrêmement fréquents, ont la faveur des milieux professionnels: marketing, discount, mix détail, mix promotionnel, rack-jobber (pour grossiste gérant de rayon), scanner (pour lecteur optique, lecteur de codes-barres, scanner, escáner, lector de código de barras, lector óptico), le verbe scanner (pour numériser, escánear), cash-and-carry (payer-prendre, commerce de gros en libre-service, paga y llevátelo, pague y lleve, cash-and-carry), couponing (cuponeo), shopping (hacer compras, ir de compras o shopping), merchandising (marchandisage, mercadeo, mercadear, comercialización), leasing (arrendamiento), zoning (zonage, zonificación), FIFO ou first-in-first-out (méthode de l'épuisement successif, primero en entrar, primero en salir), mailing (enviar por correspondencia, enviar por correo), produits me-too (imitations de produits leader, productos me-too).

- Les calques, forme de démarcation mitigée par rapport à l'anglo-américain. Les cas de francisation des mots anglais sont fréquents, comme le «mastère» (de l'anglais master, pour désigner le programme de maîtrise en administration), le «discompte» (de l'anglais discount pour désigner toutes les formes de bas prix, descuento (mot qui appartient à la langue espagnole depuis longtemps); et aussi les traductions comme «tueur de catégorie» (pour category killer, asesino de categoría), «vente de trottoir» (au lieu de braderie, pour sidewalk sale, venta callejera), «prime dans l'emballage» (pour prime in pack, premio en el paquet).

\section{Le plus ou moins grand souci de respecter les formes linguistiques propres à la langue nationale}

Mots formés sur les racines gréco-latines: mini (miniboutique, minitienda, minimall, mini centro comercial, minimarge); micro (microcircuit, microcircuito, micromarché, micromercado); super (supermarché, supermercado, superspécialiste, superespecialista); maxi (maxidiscompte, maxidescuento); hyper (hypérette, hypercentre); méga (mégavente, megaventa, mégaplex, megaplex, mégasupermarché, megasupermercado, mégacentre commercial, mega centro comercial, mégalibrairie, megalibrería); cyber (cyberconsommateur, ciberconsumidor ou cyberconsumidor, cybercommerce, cibercomercio ou cybercomercio, cyberlibrairie, ciberlibrería, cyberbilletterie, cibertaquilla). 
- Autres formes de création lexicale, telles que les mots valises: magalogue (de magazine + catalogue), spécialogue (de spécialisé + catalogue, en espagnol católogo especializado)

- Métaphores, empruntées au domaine de la marine (gondole, góndola, îlot, isla, magasin phare, l'anglais anchor qui devient locomotive, locomotora); ou au domaine animalier (en anglais, dogproduct, perro, cash cow product, vache à lait, vaca lechera, loansharking, requin de la finance, tiburón)

- le phénomène de la métonymie, comme l'usage de la couleur pour désigner la chose (produits blancs, línea blanca).

- Abréviations, fréquentes en français, mais dont l'usage arrive souvent mal à se fixer: $\mathrm{GAB}$ (guichet automatique bancaire), DAD (distributeur automatique de billets), GB (guichet bancaire), $\mathrm{CA}$ (caisse automatique) et même $\mathrm{AB}$ (automate bancaire) (sic).

Ainsi, le Dictionnaire codifiant tous ces lexèmes et toutes ces évolutions devient un témoin des formes vivantes du langage, à différents moments de son histoire et en réponse à différents critères socioculturels. «Miroir brisé, il reflète l'image temporelle d'une fonction sociale élémentaire, celle qui, articulant par le langage la pensée et l'affect, aboutit au sentiment communautaire» (Rey 1986: 625-626).

\section{Conclusion}

Nous avons voulu montrer que les mots sont le reflet des usages et des cultures, présentes et passées. Si la mémoire intime de chaque culture est déjà enfouie dans son lexique, à plus forte raison, elle l'est dans le dictionnaire, «lieu prométhéen par excellence», dit Jean Pruvost. Le dictionnaire est bien un objet du patrimoine culturel: les usages actuels se sont constitués par un cheminement historique; certains usages aujourd'hui abandonnés sont inscrits dans l'histoire des idées, des mœurs, des métiers. C'est bien là l'objectif d'un dictionnaire, enrichir le présent des richesses du passé, et ajouter aux richesses du passé les réalités du présent.

\section{NOTES}

1. L'information contenue dans cet article a été présentée dans ses grandes lignes lors du congrès tenu à Córdoba, Congreso internacional «Patrimonio Cultural», en mai 2002.

2. Nous tenons à remercier notre auxiliaire de recherche Marjorie Agrifoglio qui a fourni les équivalents espagnols des mots traités dans cet article, ainsi que le CRSH, qui a financé cette recherche.

3. Nora définit ainsi les lieux de mémoire: «à tous les sens du mot, du plus matériel et concret $[\ldots]$ au plus abstrait et intellectuellement construit [...] Depuis les chroniques de Saint-Denis, au XIII ${ }^{\mathrm{e}}$ siècle, jusqu'au Trésor de la langue française, encore inachevé; en passant par le Louvre, La Marseillaise et l'encyclopédie Larousse» (1984: VII).

4. La notion de trésor apparaît à la Renaissance, en France, avec le Thesaurus de Robert Estienne (1523), qui est en fait un dictionnaire français-latin. Par la suite; la métaphore du trésor disparaît, occultée par la conception du dictionnaire de la norme, du bon usage. Le Dictionnaire de l'Académie française se veut guide du bien dire et non pas lieu de mémoire.

5. L'espagnol, l'allemand et l'italien sont progressivement intégrés dans la base de données et feront l'objet d'éditions futures.

6. Le mot «chaland» vient du verbe ancien «chaloir» qui voulait dire s'intéresser à (contr.: nonchalant). On retrouve le radical dans les termes modernes achalandage, zone de chalandise.

\section{RÉFÉRENCES}

Benoun, M. et M.-L. Héliès-Hassid (1995): Distribution: Acteurs et stratégies, 2e éd., Paris, Économica. 
De Bessé, B. (2000) : «Le domaine», dans Béjoint, H. et P. Thoiron, Le sens en terminologie, Lyon, Presses universitaires de Lyon p. 182-197.

DANCette, J. et C. Réthoré (1997): «La mémoire du commerce: aspects étymologiques et lexicométriques», AUPELF-UREF, Ve Journées scientifiques, pp. 239-249.

Dancette, J. et C. Réthoré (2000) : Dictionnaire analytique de la distribution / Analytical Dictionary of Retailing, Montréal, Presses de l'Université de Montréal.

Meschonnic, H.(1991): Des mots et des mondes; dictionnaires, encyclopédies, grammaires, nomenclatures, Paris, Hatier.

Mirowski, P. (ed.) (1994): Natural Images in Economic Thought: Markets Read in Tooth and Claw. Cambridge/New York: Cambridge University Press. Coll. Historical Perspectives on Modern Economics.

Nora, Pierre (dir.) (1984) Les lieux de mémoire, tome I, Paris, Gallimard.

Pruvost, J. (2000): Dictionnaires et nouvelles technologies, Paris, PUF.

Rey, A. (1986): «Les trésors de la langue» in Les lieux de mémoire, tome II, Paris, Gallimard. p. 625-645.

\section{ANNEXE}

Fiche espagnole

\section{AUCTION SALE, AUCTION, SALE BY AUCTION SUBASTA, VENTA EN PÚBLICA SUBASTA}

\section{Definición}

Proceso de venta pública de bienes o títulos de propiedad al mejor postor.

\section{Precisiones}

La subasta se realiza en voz alta en una sala de venta (auction room). El subastador (auctioneer) presenta el artículo, pide una primera oferta o puja, (bid) o anuncia él mismo un precio inicial mínimo. Los compradores potenciales compiten ofreciendo cada vez más por el producto y, a la cuenta de tres, el subastador baja el martillo y adjudica (knock down, strike off) el artículo al mejor postor (bidder). Si éste no puede pagar la suma ofrecida o ha hecho una oferta falsa (false bidding), se declara la quiebra de la subasta y se adjudica el bien al segundo postor o se subasta de nuevo.

\section{Nociones relacionadas}

remate; licitación; oferta de concurso o de subasta; propuesta; subasta a viva voz o a la llana; subasta por pliego cerrado

\section{Contexto}

La celebración de una pública subasta consiste en ofertar (...) la venta de un bien a favor de quien ofrezca, mediante el sistema de pujas y dentro del plazo concedido al efecto, el precio más alto por encima de un mínimo, ya se fije éste inicialmente o mediante ofertas descendentes realizadas en el curso del propio acto (Tornos, J. et al. (1996): Ordenación del comercio minorsita, Barcelona, Editorial Praxis, p. 394). 